\title{
Acute Disseminated Encephalomyelitis Responsive to Cyclophosphamide Therapy: A Case Report
}

\author{
Joshua Rothenberg $^{\mathrm{a}, \mathrm{b}}$, Angie Lastra ${ }^{\mathrm{a}}$, Gemayaret Alvarez ${ }^{\mathrm{a}}$, Robert Irwin ${ }^{\mathrm{a}}$
}

\begin{abstract}
Acute disseminated encephalomyelitis (ADEM) is a rare neurologic condition that affects the central nervous system (CNS). In this report, a 22-year-old fraternal twin male developed ADEM 6 months post viral infection. He presented non-verbal with doll's eyes, a right facial droop, right-sided upper and lower extremity weakness, as well as brisk reflexes and clonus on the right. He failed to improve with traditional therapies such as intravenous steroids, intravenous immunoglobulin, and plasmapheresis. However, 2 days following a 3-day course of intravenous cyclophosphamide $1,000 \mathrm{mg} / \mathrm{m}^{2}$, he spontaneously opened his eyes, developed masticatory movements, mouthed words, and regained movement in his extremities against gravity. There was a $40.7 \%$ and $16.6 \%$ decrease in lesion area in brain magnetic resonance imaging (MRI) T2 FLAIR sagittal and axial images, respectively over that 2-day period. This is the first reported case that shows neurological improvement, measured by the functional independence measure (FIM) scale, in addition to decreased lesion size on brain MRI T2 FLAIR images after implementation of cyclophosphamide therapy. Cyclophosphamide should be considered as an alternative therapy in patients with refractory ADEM.
\end{abstract}

Keywords: Brain; Cyclophosphamide; Encephalomyelitis; Acute disseminated; Humans; Immunoglobulins; Intravenous; Magnetic resonance imaging; Plasmapheresis; Twins; Dizygotic

\section{Introduction}

Acute disseminated encephalomyelitis (ADEM) is an acute demyelinating disorder affecting the white matter of the cen-

Manuscript accepted for publication May 11, 2015

aPhysical Medicine and Rehabilitation Residency at University of Miami Leonard M. Miller School of Medicine, Jackson Memorial Hospital, Miami, FL, USA

${ }^{\mathrm{b} C}$ Corresponding Author: Joshua Rothenberg, Physical Medicine and Rehabilitation Residency at University of Miami Leonard M. Miller School of Medicine, Jackson Memorial Hospital, PO Box 016960 (D-461), Miami, FL 33101, USA. Email: jrothenberg12786@gmail.com

doi: http://dx.doi.org/10.14740/jmc2176w tral nervous system (CNS) [1]. Although no specific etiology has been correlated, there appears to be an association with a history of antecedent vaccination or infection [2]. The suspected pathophysiology is through an autoimmune process via molecular mimicry. Clinical symptoms include visual loss, fatigue, malaise, headache, nausea, and myalgias [3], with the hallmark feature being focal or multifocal central nervous system effects that occur relatively rapidly [1]. CNS effects range from lethargy to coma, hemiparesis or paraparesis, and/ or cranial nerve palsies [4]. Recovery varies ranging from days to more commonly weeks to months [1].

The incidence of ADEM has been estimated to be 0.4 per 100,000 population per year [5]. The disease is more commonly found in children and young adults [2]. Diagnosis includes looking at CSF, EEG, and magnetic resonance imaging (MRI) on T2-weighted images with FLAIR [6]. CSF may look normal but often shows increased pressure, raised protein, and sometimes may present with increased gammaglobulin $(\operatorname{IgG})$ and myelin basic protein. MRI, the best test, looks for demyelinating lesions throughout the posterior fossa and cerebral hemispheres [1]. The lesions will appear as patchy infiltrates on a T2-weighted signal MRI with FLAIR [6]. The accepted standard therapy at this time is high-dose intravenous corticosteroids as a first-line therapy. When refractory, the main alternative is IVIG followed by plasmapheresis. When these treatments fail, cytotoxic drugs have been used as a last line agent [1]. Schwarz et al reported symptom improvement but not radiologic improvement in ADEM patients post-cyclophosphamide. This is the first reported case that shows improvement in both neurologic symptoms and brain MRI after cyclophosphamide therapy. The role medications and rehabilitation played in this patient's recovery will be discussed.

\section{Case Report}

A 22-year-old fraternal twin male, with a significant history of upper respiratory infection 6 months prior, presented to a community hospital with right upper and lower extremity numbness and weakness, difficulty with speech, and new onset urinary retention. A lumbar puncture performed showed a WBC count of $24 / \mathrm{mm}^{3}$, protein of $78 \mathrm{mg} / \mathrm{dL}$, and glucose of $97 \mathrm{mg} / \mathrm{dL}$ with positive oligoclonal bands. IgG synthesis was elevated at 7.2 and CSF cytology showed abundant lymphocytes and mono- 


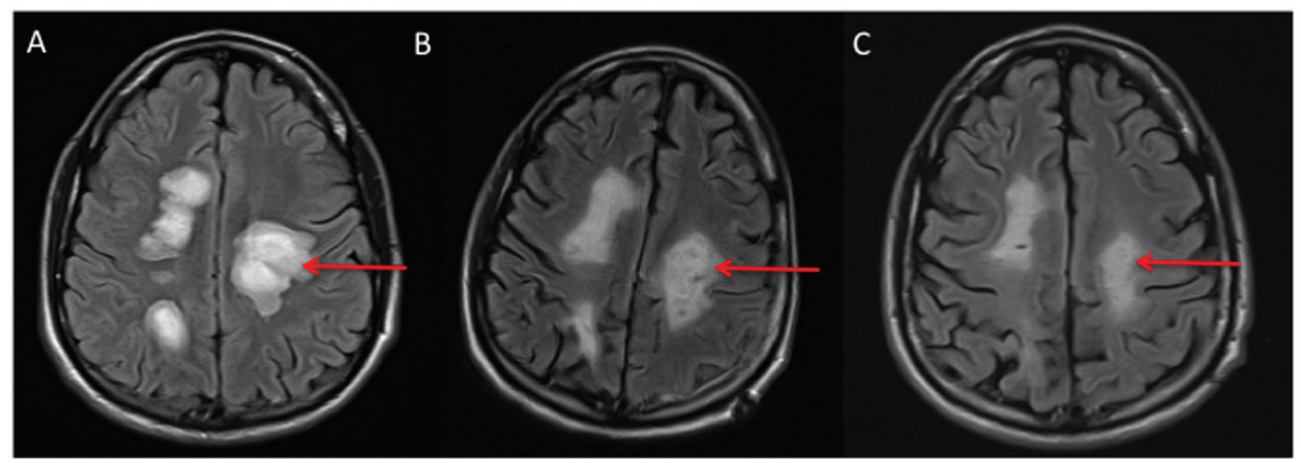

Figure 1. Comparison of brain MRI T2 FLAIR axial images demonstrating hyperintense areas of demyelination. (A) Four hyperintense demyelinating lesions, a total sum area of $21.381 \mathrm{~cm}^{2}$, upon admission to hospital. (B) Three demyelinating lesions with decreased hyperintensity and total sum area of $17.839 \mathrm{~cm}^{2}$, a $16.6 \%$ decrease in area compared to (A) 2 days after receiving a 3-day course of IV cyclophosphamide $1,000 \mathrm{mg} / \mathrm{m}^{2}$. (C) Three demyelinating lesions with further decreased hyperintensity and total sum area of $8.434 \mathrm{~cm}^{2}$, a $60.6 \%$ decrease in total sum area compared to (A) 2 months after receiving cyclophosphamide.

cytes. MRI showed multiple oval areas of signal alteration within periventricular deep and subcortical white matter of the brain on T2 FLAIR sequences. The patient was treated with IV methylprednisolone $1 \mathrm{~g}$ daily for 5 days. He showed no improvement in his function, so this was followed by IVIG for 5 days. Again no improvement was demonstrated, so he was given four treatments of plasmapheresis and continued without improvement.

Due to the patient's lack of response to IV steroids, IVIG, and plasmapheresis, he was transferred to a university-based hospital. Physical examination upon admission demonstrated the patient was neurologically compromised; he presented with a global aphasia, non-purposeful movements, and posturing in response to painful stimuli. Additionally, he exhibited a right facial droop and inconsistent eye opening to verbal stimulation. Upper motor neuron signs were also prevalent; his muscle tone was spastic, with a grade of three out of four on the modified Ashworth scale in his right upper extremity flexors and right lower extremity extensors. Furthermore, his deep tendon reflexes were brisk and he demonstrated a sustained clonus on the right lower extremity. Brain MRI upon admission showed worsening and interval progression of multiple areas of abnormal T2 FLAIR signal intensity with patchy mixed solid, fluffy, and ring-like enhancement in the white matter of the infra- and supratentorial brain (Fig. 1, 2, Table 1). ADEM was favored as the diagnosis (Fig. 3).

The patient remained in a minimally conscious state despite all the treatments. He could open his eyes, but could not follow commands. His hospital course was complicated by multiple UTIs, sepsis, as well as a Clostridium difficile infection. An EEG was performed which showed generalized theta slowing, suggestive of diffuse cerebral dysfunction. A left-sided craniectomy and open biopsy of the left parietal lobe lesion was completed and showed demyelination with gemistocytic astrocytosis and macrophage infiltration.

Given the patient's lack of improvement, the patient completed a truncated 3-day course of IV cyclophosphamide 1,000 $\mathrm{mg} / \mathrm{m}^{2}$ (Cytoxan, Princeton, $\mathrm{NJ}$ ) that was discontinued due to evolving neutropenia. On day 2 of the cyclophosphamide treatment, the patient was noted to have intermittent eye contact, and some return of motor control, as exhibited by mouthing words, and moving his toes. Follow-up brain MRI showed mild overall improvement in the size and extent of multifocal patchy areas of signal hyperintensity involving both cerebral hemispheres and brain stem on the T2 FLAIR sequences (Fig. 1, 2,

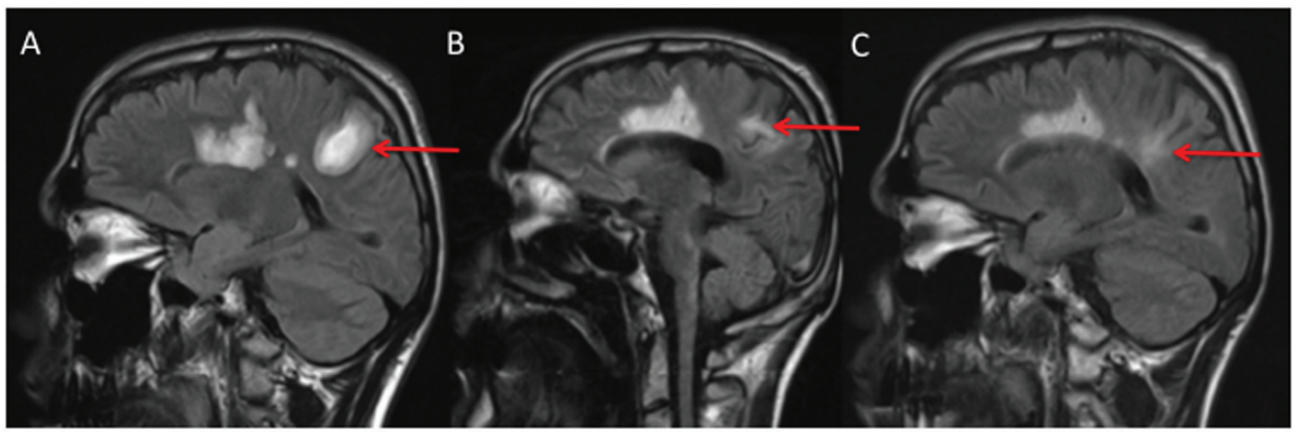

Figure 2. Comparison of brain MRI T2 FLAIR sagittal images demonstrating hyperintense areas of demyelination. (A) Three well demarcated hyperintense demyelinating lesions, a total sum area of $12.291 \mathrm{~cm}^{2}$, upon admission to hospital. (B) Two demyelinating lesions with decreased hyperintensity and total sum area of $7.286 \mathrm{~cm}^{2}$, a $40.7 \%$ decrease in area compared to (A) 2 days after receiving a 3-day course of IV cyclophosphamide $1,000 \mathrm{mg} / \mathrm{m}^{2}$. (C) Two demyelinating lesions with further decreased hyperintensity and total sum area of $4.732 \mathrm{~cm}^{2}$, a $61.5 \%$ decrease in total sum area compared to (A) 2 months after receiving cyclophosphamide. 
Table 1. Comparison of Total Sum Area and \% Change of Lesion Area Pre- and Post-Cyclophosphamide Therapy

\begin{tabular}{lllll}
\hline Brain MRI T2 FLAIR series & $\begin{array}{l}\text { Total sum } \\
\text { area }\left(\mathbf{c m}^{\mathbf{2}}\right)\end{array}$ & $\begin{array}{l}\text { \% Change compared to } \\
\text { pre-cyclophosphamide }(\mathbf{\%})\end{array}$ & $\begin{array}{l}\text { Total sum } \\
\text { area }\left(\mathbf{c m}^{2}\right)\end{array}$ & $\begin{array}{l}\text { \% Change compared to } \\
\text { pre-cyclophosphamide }(\mathbf{\%})\end{array}$ \\
\hline Pre-cyclophosphamide & 12.291 & 0 & 21.381 & 0 \\
Two days post-cyclophosphamide & 7.286 & 40.7 & 17.839 & 16.6 \\
Two months post-cyclophosphamide & 4.732 & 61.5 & 8.434 & 60.6 \\
\hline
\end{tabular}

Table 1). Over the next 3 weeks, he became more responsive and was sent to inpatient rehabilitation 3 months after his initial presentation. He showed a 10-point improvement on the functional independent measure (FIM) scale dating from hospital admission to inpatient rehabilitation admission, a 2-month time frame (Table 2).

A repeat brain MRI upon arrival to the rehabilitation unit showed interval improvement in the size and extent of multiple patchy confluent areas of hyperintense FLAIR signal abnormality within the periventricular and subcortical white matter (Fig. 1, 2, Table 1).
Upon discharge, 6 months after initial presentation, the patient spontaneously moved his extremities, responded appropriately with speech, ate full meals, tracked with his eyes, and walked 150 feet with bilateral AFOs and a hemiwalker. He displayed progress in comprehension, expression, social interaction, problem solving, and memory. He exhibited improvement in speed of process, such as reaction time, improved insight, and increased initiative. He was discharged after 2.5 months of inpatient rehabilitation, improving a total 32 points on the FIM scale (Table 2). He continued therapy in an outpatient rehabilitation setting.

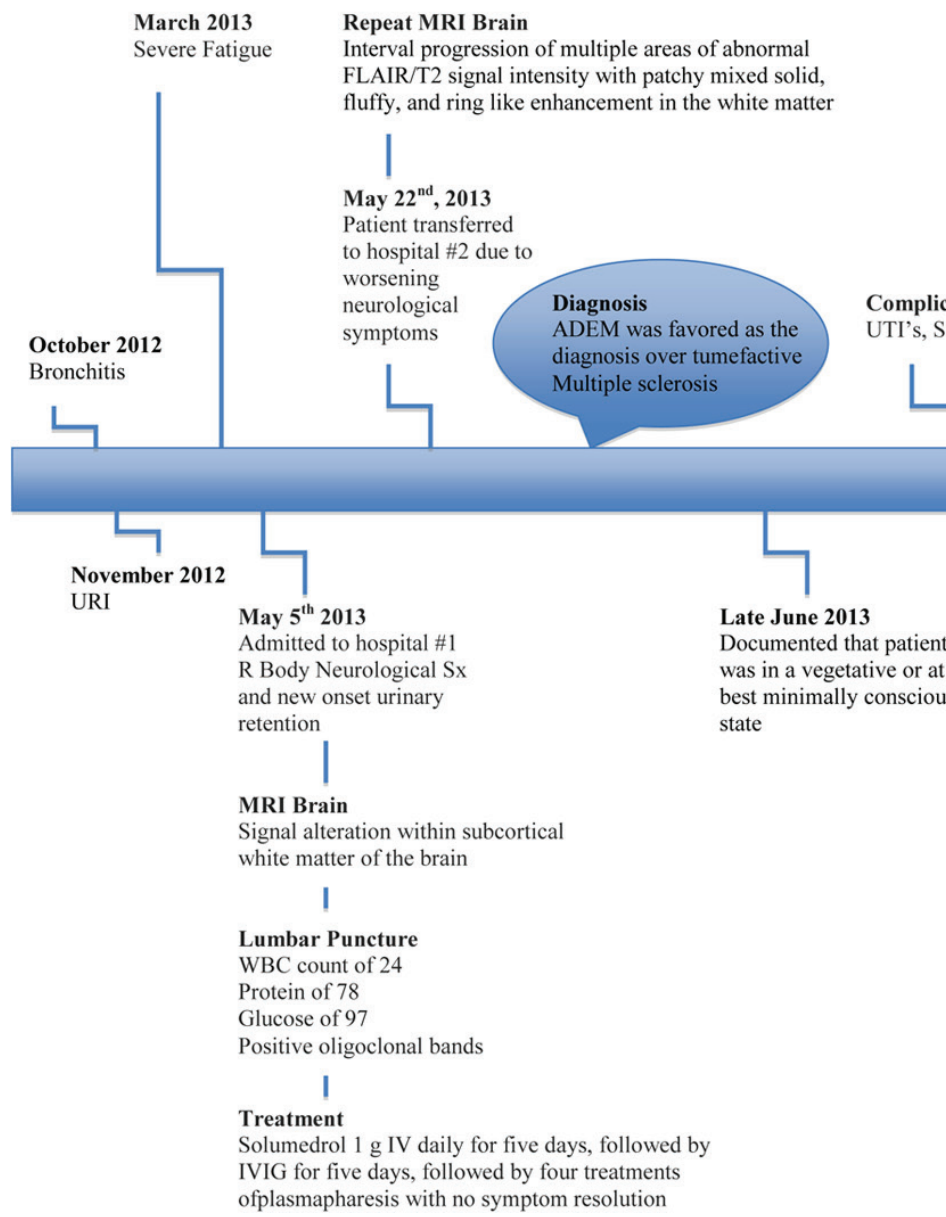

September $9^{\text {th }}, 2013$

Repeat MRI Brain

Continued interval improvement in the size and extent of hyperintense FLAIR signal

FLAIR/T2 signal intensity with patchy mixed solid,

and ring like enhancement in the white matter

uly $3^{\text {rd }}, 2013$

Three-day course of

Cyclophosphamide that

was stopped due

toneutropenia, in

conjunction with a five-day

course of IV Solumedrol
Repeat MRI B

Less Gadolinium

enhancement of

lesions
August 9th, 2013 October $24^{\text {th }}, 2013$

Inpatient rehabilitation
I's, Sepsis, C.Diff 
Table 2. Comparison of Functional Independence Measure (FIM) Score Ratings on Hospital Admission, Inpatient Rehab Admission, and Inpatient Rehab Discharge

\begin{tabular}{|c|c|c|c|}
\hline FIM categories & $\begin{array}{l}\text { FIM scores on hospital } \\
\text { admission (May 22, 2013) }\end{array}$ & $\begin{array}{l}\text { FIM scores on rehabilitation } \\
\text { admission (August 9, 2013) }\end{array}$ & $\begin{array}{l}\text { FIM scores on rehabilitation } \\
\text { discharge (October 22, 2013) }\end{array}$ \\
\hline Eating & 1 & 1 & 5 \\
\hline Grooming & 1 & 1 & 5 \\
\hline Bathing & 1 & 1 & 3 \\
\hline Upper body dressing & 1 & 1 & 4 \\
\hline Lower body dressing & 1 & 1 & 1 \\
\hline Toileting & 1 & 1 & 1 \\
\hline Bladder management & 1 & 1 & 2 \\
\hline Bowel management & 1 & 1 & 1 \\
\hline Bed to chair transfer & 1 & 1 & 4 \\
\hline Toilet transfer & 1 & 1 & 3 \\
\hline Tub transfer & 1 & 1 & 3 \\
\hline Locomotion & 1 & 1 & 5 \\
\hline Stairs & 1 & 1 & 1 \\
\hline Cognitive comprehension & 1 & 4 & 5 \\
\hline Expression & 1 & 4 & 5 \\
\hline Social interaction & 1 & 3 & 5 \\
\hline Problem solving & 1 & 2 & 3 \\
\hline Memory & 1 & 2 & 4 \\
\hline Total & 18 & 28 & 60 \\
\hline
\end{tabular}

\section{Discussion}

ADEM is an uncommon, acute CNS demyelinating disorder [1]. The patient's diagnosis was based on clinical presentation, imaging, CSF analysis, as well as brain biopsy findings. Two characteristics that make this case unique are explored. These include the time from the infection to the onset of symptoms, and his responses to the different therapies initiated.

A variable that is commonly linked to ADEM is a preceding viral infection or prior immunization. According to Gulay et al, ADEM classically occurs within 2 days to 4 weeks following a viral infection. In fact, they state approximately $70-93 \%$ of patients report a clinically evident antecedent infection during the prior few weeks [7]. Additionally, Murthy et al showed $72 \%(13 / 18)$ had an upper respiratory infection on an average of 2 days to 4 weeks prior to presentation, with an average of 10 days prior to onset of neurological symptoms [8]. However, this case highlights an adult male with past history significant for viral infection 6 months prior to the development of neurological deficits, nearly five times greater than the expected preceding time frame, and out of the standard acute window.

The treatment of ADEM is targeted to suppress a presumed aberrant immune response. Intravenous corticosteroids are widely accepted as the first-line treatment for acute episodes [7]. Approximately, two-thirds of patients respond to this regimen. The chief alternative therapy is IV immunoglobulin or plasmapheresis. In some cases, cytotoxic agents have been used with success [1]. At this time, there have been no randomized controlled trials for the treatment of ADEM.

In this case, the patient not only failed to respond but also had neurologic worsening after receiving IV steroids, IVIG and plasmapheresis, and thus was given a poor prognosis. After 2 months, refractory to standard therapies, and having excluded CNS infection and malignancy, he received a truncated 3-day course trial of cyclophosphamide, normally given as a 5-day course, due to leukopenia. Within 1 week of this regimen, there was a significant improvement in patient's neurological status, function, and brain MRI findings.

Only one published article during the literature search, by Schwarz et al, discussed ADEM, and cyclophosphamide as an alternative and effective therapy for refractory and severe cases [9]. However, even within this publication, sample size and data were limited for those that received cyclophosphamide. It is worth mentioning that spontaneous recovery might have influenced the patient's improvement, but it is believed that its close proximity to the addition of cyclophosphamide was more crucial. This is the first reported case of ADEM to show clear improvement in symptoms and imaging after initiation of cyclophosphamide.

\section{Conclusion}

Significant functional and neurological improvements were 
demonstrated in this case of ADEM. An immunosuppressive agent, cyclophosphamide, should be considered in the future as an alternative treatment in patients with ADEM refractory to standard therapies. This patient's neurological recovery was significant after addition of cyclophosphamide to the management plan, which was shown objectively on brain imaging. Additionally, the patient showed great functional improvement in cognitive tasks, ambulation status, and activities of daily living evidenced by his progression on the FIM score rating. This lends support that inpatient rehabilitation when combined with an appropriate medication regimen improves functional and neurological outcomes of patients with ADEM.

\section{Acknowledgement}

Dr. Kester Nedd, DO, and Dr. Kottil Rammohan, MD provided contributions to this case.

\section{Disclosure}

There were no funding or grants or equipment provided for the project from any source nor any financial benefits to the authors. This case abstract was presented as a poster at the American Academy of Physical Medicine and Rehabilitation Conference, on November 12 - 16, 2014, at San Diego Convention Center, San Diego, CA.

\section{References}

1. Garg RK. Acute disseminated encephalomyelitis. Post- grad Med J. 2003;79(927):11-17.

2. Hynson JL, Kornberg AJ, Coleman LT, Shield L, Harvey AS, Kean MJ. Clinical and neuroradiologic features of acute disseminated encephalomyelitis in children. Neurology. 2001;56(10):1308-1312.

3. Hartung HP, Grossman RI. ADEM: distinct disease or part of the MS spectrum? Neurology. 2001;56(10):12571260.

4. Modi G, Mochan A, Modi M, Saffer D. Demyelinating disorder of the central nervous system occurring in black South Africans. J Neurol Neurosurg Psychiatry. 2001;70(4):500-505.

5. Leake JA, Albani S, Kao AS, Senac MO, Billman GF, Nespeca MP, Paulino AD, et al. Acute disseminated encephalomyelitis in childhood: epidemiologic, clinical and laboratory features. Pediatr Infect Dis J. 2004;23(8):756764.

6. Bizzi A, Ulug AM, Crawford TO, Passe T, Bugiani M, Bryan RN, Barker PB. Quantitative proton MR spectroscopic imaging in acute disseminated encephalomyelitis. AJNR Am J Neuroradiol. 2001;22(6):1125-1130.

7. Alper G, Heyman R, Wang L. Multiple sclerosis and acute disseminated encephalomyelitis diagnosed in children after long-term follow-up: comparison of presenting features. Dev Med Child Neurol. 2009;51(6):480486.

8. Murthy SN, Faden HS, Cohen ME, Bakshi R. Acute disseminated encephalomyelitis in children. Pediatrics. 2002;110(2 Pt 1):e21.

9. Schwarz S, Mohr A, Knauth M, Wildemann B, StorchHagenlocher B. Acute disseminated encephalomyelitis: a follow-up study of 40 adult patients. Neurology. 2001;56(10):1313-1318. 